\title{
Piezoelectric Transducers Applied in Structural Health Monitoring: Data Acquisition and Virtual Instrumentation for Electromechanical Impedance Technique
}

\author{
Fabricio Guimarães Baptista and Jozue Vieira Filho \\ Department of Electrical Engineering, Sao Paulo State University, Ilha Solteira, \\ Brazil
}

\section{Introduction}

This chapter reports the application of piezoelectric transducers in the detection of structural damage, focusing on the data acquisition based on virtual instrumentation for the appropriate analysis of the signals from these transducers, allowing fast and accurate measurements.

There is a growing interest in systems able to continuously monitor a structure and timely detect incipient damage, ensuring a high level of safety and reducing maintenance costs. This concept is commonly known as Structural Health Monitoring (SHM) in the literature. Many techniques can be used to develop SHM systems, but the electromechanical impedance (EMI) technique has the advantage of using small and very thin piezoelectric patches, with thickness on the order of a few tenths of millimeters. Thus, these devices are like adhesives bonded to the monitored structure, allowing a large area of the structure to be monitored with negligible effects on its mechanical properties. Due to the piezoelectric effect, when a piezoelectric transducer is bonded to the structure to me monitored, there is an interaction between the mechanical impedance of the host structure and the electrical impedance of the transducer. Therefore, changes in the mechanical impedance of the host structure caused by damage, such as cracks or corrosions, can be detected simply by measuring the electrical impedance of the transducer in a suitable frequency range, which is easier to measure than the mechanical impedance.

Usually, the measurement of the electrical impedance of piezoelectric transducers, basic stage of the technique, is carried out by commercial impedance analyzers. Although accurate, these instruments are expensive, bulky and slow for real-time SHM applications, where fast measurements from multiple sensors are required. According to the results presented here, the virtual instrumentation can be a great ally in the development of realtime SHM systems in structures with large number of piezoelectric transducers. Measurement systems based on virtual instrumentation are fast and extremely versatile, allowing adjustments to be easily incorporated in accordance with the user needs. 
This chapter is organized as follows. A brief introduction to SHM is presented in Section 2, indicating its importance, the main fields of application and the main methodologies involved. The EMI technique is discussed in Section 3. In this section, an equivalent electromechanical circuit is obtained to relate the electrical impedance of the transducer to the mechanical impedance of the monitored structure. Measurements systems based on virtual instrumentation are studied in Section 4. Two measurement methods are analyzed: frequency domain and time domain measurement. Finally, the chapter concludes with Section 5 citing the most relevant points.

\section{Structural health monitoring (SHM)}

This section is based on the literature review conducted by Sohn et al. (2004) from Los Alamos National Laboratory. The SHM systems are designed to continuously monitor a structure and to detect incipient structural damage. According to Rytter (1993), in advanced systems there is a five-step process to be followed in the characterization of damage: (1) damage detection; (2) location of damage in the structure; (3) determining which type of damage is present; (4) estimate its severity; (5) analysis of the remaining useful life of the structure, i.e., the prognosis.

Among the various fields of application, we can cite the large civil infrastructures (bridges, buildings, roads, oil rigs, etc.), the aeronautical and aerospace structures (aircraft, helicopters, satellites, space stations, etc.), and large marine structures (submarines and ships). There are both scientific and economic motivations for the use of an SHM system. From the scientific point of view, the monitoring and detection of structural damage mean to achieve a high level of safety. From the economic point of view, systems with this capability allow a significant reduction in maintenance costs. For example, Cawley (1997) suggested the use of an SHM system to identify corrosion in pipelines of chemical and petrochemical industries, in which the costs associated with the removal of these pipelines for inspection is prohibitive. The Federal Highway Administration estimates that nearly 35\% of all bridges in the United States are either structurally or functionally deficient (Wang et al., 1997). The cost of repair or rebuilding lies in the billions of dollars Therefore, SHM systems could reduce this cost while providing high level of safety for users during repair or assessment.

Currently, the aviation industry is one of most focused fields of application. Although the design and criterions for certification of an aircraft already guarantee a high level of security, an SHM system could significantly reduce the repair and maintenance costs, which represent $27 \%$ of the cost of its life cycle (Kessler et al., 2002). The direct costs related to the repair could be reduced by detecting damage in an early stage. In addition, the indirect costs could be reduced by a lower frequency at which the aircraft would be shut down for maintenance.

The definition of damage is important in SHM systems. Damage is any change in the structure that may affect its performance and safety. Implicit in this definition is the concept that damage detection is based on the comparison between two states of the structure. In a first state, the structure is considered healthy and it is used as a reference for comparison with an updated state after a probable occurrence of damage. Damage such as cracks and corrosion can change various properties of the monitored structure as, for example, mass, 
stiffness, energy dissipation, mechanical impedance and its cross-section area. In addition, these changes alter the dynamic properties of the structure. From this concept, there are the techniques based on the frequency response function (FRF). In these techniques, the data for the evaluation of the structure are collected while the structure is in dynamic condition using one of two methods: ambient excitation and forced-excitation. In the ambient excitation, the vibration produced by the structure during its normal operation is utilized. The vibration of a bridge due to the vehicular traffic is an example of ambient excitation. On the other hand, the forced-excitation is controlled using actuators, such as shakers and piezoelectric transducers.

There are many techniques for developing SHM systems. Several techniques are based on non-destructive evaluation (NDE) methods, such as acoustic emission, comparative vacuum, magnetic particle inspection, Eddy current, and methods based on optical fibre sensors. In this chapter, we aim to analyze the electromechanical impedance (EMI) technique, which is a method based on forced-excitation. In this technique, low-cost and thin piezoelectric patches are bonded to the host structure and combine both the functions of actuator and sensor. The EMI technique is presented in the next section.

\section{Electromechanical Impedance (EMI)}

\subsection{Basic concept}

The EMI technique is a form of nondestructive evaluation based on the FRF which has the advantages of its simplicity and using thin and low-cost piezoelectric transducers. The most widely used piezoelectric transducers are the PZT (Pb-lead Zirconate Titanate) ceramics and MFC (Macro-Fiber Composite), which have thickness on the order of a few tenths of millimeters. These characteristics make the EMI technique especially attractive for the monitoring of aircraft structures, which is one of the most prominent application fields nowadays. The EMI technique is the only PZT-based technique that has characteristics for the development of a real-time and in-situ SHM system in aircrafts (Gyekenyesi et al., 2005). These devices are bonded to the structure to be monitored through a high-strength adhesive that can be instantaneous glue based on cyanoacrylate or epoxy resin. Due to the piezoelectric effect, there is a relationship between the mechanical properties of the structure and the electrical impedance of the transducer. Therefore, it is possible to monitor variations in these mechanical properties by measuring the electrical impedance. For example, Figure 1 shows a PZT patch from Piezo Systems bonded to an aluminum beam.

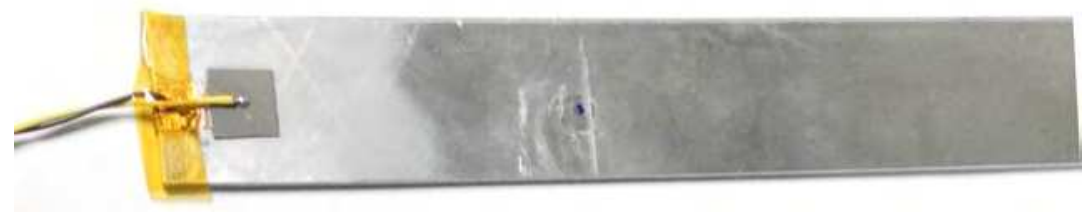

Fig. 1. A PZT patch bonded to an aluminum beam for damage detection.

In Figure 1, both sides of the PZT ceramic are coated by a thin metal layer, where wires are soldered to connection. Through these wires, the patch is excited and, simultaneously, its electrical impedance is measured in an appropriate frequency range. As a result, these 
ceramics combine the functions of sensor (direct piezoelectric effect) and actuator (reverse piezoelectric effect.

In order to obtain the relationship between the electrical impedance of the transducer and the mechanical impedance of the structure, we should analyze the wave propagation in the structure when the transducer is excited. For this analysis we consider the representation of a square PZT patch bonded to a host structure, as shown in Figure 2.

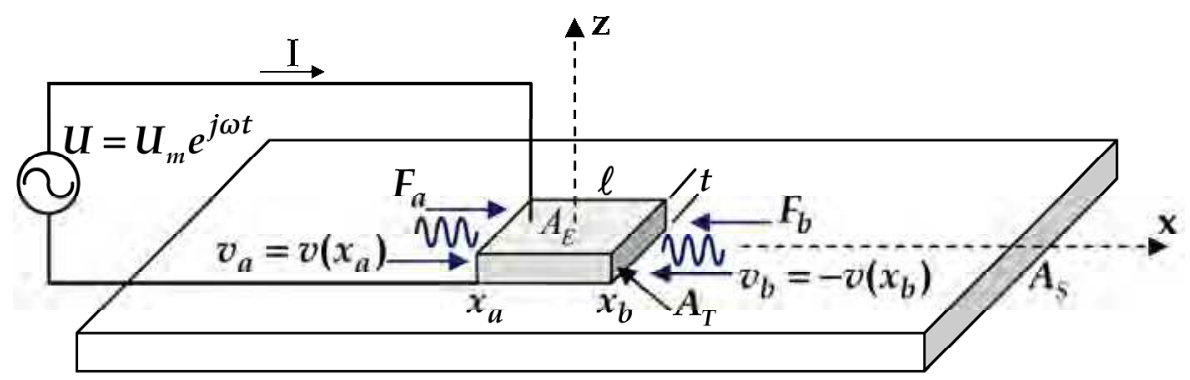

Fig. 2. Principle of the EMI technique; a square PZT patch is bonded to the structure to be monitored.

In Figure 2, a square PZT patch with side $\ell$ and thickness $t$ is bonded to a rectangular structure with cross-sectional area $A_{S}$, which is perpendicular to the direction of its length. An alternating voltage $U$ is applied to the transducer through the bottom and top electrodes, and the response is a current with intensity I. If the PZT patch has small thickness, a wave propagating at velocity $v_{a}$ in the host structure reaches the patch side with coordinate $x_{a}$ and surface area $A_{T}$ causing the force $F_{a}$. Similarly, in the side of coordinate $x_{b}$ there is a force $F_{b}$ due to the incoming wave propagating at velocity $v_{b}$.

To find an equivalent circuit that represents the behavior of the PZT patch bonded to the structure, we need to determine the relationship between the mechanical quantities $\left(F_{a}, F_{b}\right.$, $\left.v_{a}, v_{b}\right)$ and the electrical quantities $(U, I)$, as shown in the next section.

\subsection{Theoretical analysis}

The theory developed in this section is based on analysis presented by Royer \& Dieulesaint (2000).

As the thickness of the transducer is much smaller than the other dimensions, the deformation in its thickness direction ( $z$-axis) due to the applied electric field is negligible. In general, for PZT patches of $5 \mathrm{~A}$ and $5 \mathrm{H}$ type with thickness ranging from 0.1 to $0.3 \mathrm{~mm}$, the deformation in the thickness direction is on the order of nanometers. On the other hand, the deformations in the sides $\ell$ (transverse direction) are on the order of micrometers. Therefore, the vibration mode is predominantly transverse to the direction of the applied electric field. In addition, if the applied voltage $U$ is low in the order of a few volts and hence the resultant electric field is also low, the piezoelectric effect is predominantly linear and the non-linearities can be neglected. From this assumption and considering the class $6 \mathrm{~mm}$ for PZT ceramics (Meitzler, 1987), the basic piezoelectric equations for this case are given by 


$$
\begin{aligned}
& D_{3}=d_{31}\left(T_{1}+T_{2}\right)+d_{33} T_{3}+\varepsilon_{33}^{T} E_{3} \\
& S_{1}=s_{11}^{E} T_{1}+s_{12}^{E} T_{2}+s_{13}^{E} T_{3}+d_{31} E_{3} \\
& S_{2}=-s_{12}^{E} T_{1}-s_{11}^{E} T_{2}+s_{13}^{E} T_{3}+d_{31} E_{3}
\end{aligned}
$$

where $E_{3}$ and $D_{3}$ are the electric field and electrical displacement, respectively; $T_{1}, T_{2}$, and $T_{3}$ are the stress components; $S_{1}$ and $S_{2}$ are the strain components; $d_{31}$ and $d_{33}$ are the piezoelectric constants; $s_{11}, s_{12}$, and $s_{13}$ are the compliance components at constant electric field; $\varepsilon_{33}$ is the permittivity at constant stress. The superscripts $E$ and $T$ donate constant electric field and constant stress, respectively, and the subscripts 1, 2, and 3 refer to the directions $x, y$, and $z$, respectively.

Although the transducer is square and the deformations in both sides are approximately the same, only the deformation along the length of the structure is considered for the onedimensional (1D) assumption. Thus, the main propagation direction is considered along the length direction (x-axis) perpendicular to the cross-section area $A_{S}$ of the host structure, as shown in Figure 2. Therefore, for 1-D assumption, it is correct to consider $T_{2}=T_{3}=S_{2}=0$. Hence, the Equations (1) to (3) can be rewritten as follows

$$
\begin{aligned}
& D_{3}=d_{31} T_{1}+\varepsilon_{33} E_{3} \\
& S_{1}=s_{11} T_{1}+d_{31} E_{3}
\end{aligned}
$$

The patch is essentially a capacitor. Thus, due to the voltage source, there is a charge density $\left(\rho_{e}\right)$ on the electrodes of the patch and according to the Poisson equation we have

$$
\frac{\partial D_{3}}{\partial z}=\rho_{e}
$$

This results in a current of intensity $I_{C} e^{j \omega t}$. If the current is uniform over the entire area of the electrodes, the charge conservation requires

$$
\frac{\partial D_{3}}{\partial t}=J(t)=\frac{I_{C} e^{j \omega t}}{A_{E}}
$$

where $J(t)$ is the current density and $A_{E}$ is the area of each electrode.

It is appropriate to put the stress $T_{1}$ in function of the electric displacement $D_{3}$. So, from Equation (5) and considering the following relation

$$
S_{x}=\frac{\partial u_{x}}{\partial x}
$$

where $u_{x}$ is the displacement in the $\mathrm{x}$ direction, we can obtain

$$
T_{1}=\frac{1}{s_{11}} \frac{\partial u_{x}}{\partial x}-\frac{d_{31}}{s_{11} \varepsilon_{33}} D_{3}
$$


Differentiating Equation (9) with respect to time

$$
\frac{\partial T_{1}}{\partial t}=\frac{1}{s_{11}} \frac{\partial}{\partial x}\left(\frac{\partial u_{x}}{\partial t}\right)-\frac{d_{31}}{s_{11} \varepsilon_{33}} \frac{\partial D_{3}}{\partial t}
$$

and considering the velocity given by

$$
v=\frac{\partial u_{x}}{\partial t}
$$

and considering the charge conservation in Equation (7), we can rewrite the expression as follows

$$
\frac{\partial T_{1}}{\partial t}=\frac{1}{s_{11}} \frac{\partial v}{\partial x}-\frac{d_{31}}{s_{11} \varepsilon_{33}} \frac{I_{C} e^{j \omega t}}{A_{E}}
$$

The motion equation for this case is given by

$$
\rho_{T} \frac{\partial^{2} v}{\partial t^{2}}=\frac{1}{s_{11}} \frac{\partial^{2} v}{\partial x^{2}}
$$

where $\rho_{T}$ is the mass density of the piezoelectric material. The general solution for Equation (13) is the sum of two waves propagating in opposite directions, as shown in Figure 2. In steady state, we have

$$
v=\left(m \cdot e^{-j k x}+n \cdot e^{j k x}\right) e^{j \omega t}=\left(v_{m}+v_{n}\right) e^{j \omega t}
$$

where $m$ and $n$ are constants and $k$ is the wave number given by

$$
k=\frac{\omega}{V}
$$

where $V$ is the velocity of propagation given by

$$
V=\frac{1}{\sqrt{s_{11} \rho_{T}}}
$$

Substituting the velocity given in Equation (14) into the stress in Equation (12) and integrating with respect to time, we have

$$
\begin{gathered}
T_{1}=\frac{1}{s_{11}} \frac{\partial}{\partial x}\left(m \cdot e^{-j k x}+n \cdot e^{j k x}\right) \int e^{j \omega t} \mathrm{dt}-\frac{d_{31}}{s_{11} \varepsilon_{33}} \frac{I_{C}}{A_{E}} \int e^{j \omega t} \mathrm{dt} \\
T_{1}=-\frac{k}{\omega s_{11}}\left(m \cdot e^{-j k x}-n \cdot e^{j k x}\right) e^{j \omega t}-\frac{d_{31}}{j \omega s_{11} \varepsilon_{33}} \frac{I_{C}}{A_{E}} e^{j \omega t}
\end{gathered}
$$

The characteristic (acoustic) impedance $\left(Z_{T}^{A}\right)$ of the PZT patch is given by 


$$
Z_{T}^{A}=\frac{k}{\omega s_{11}}
$$

Substituting Equation (19) into Equation (18) and hiding the term $e^{j \omega t}$ just for simplicity, the equation of stress can be rewritten as

$$
T_{1}=-Z_{T}^{A}\left(m \cdot e^{-j k x}-n \cdot e^{j k x}\right)+\mathrm{j} \frac{d_{31}}{s_{11} \varepsilon_{33}} \frac{I_{C}}{\omega A_{E}}
$$

The forces acting on each face of the transducer can be calculated by

$$
\begin{aligned}
& F_{a}=-A_{T} T_{1}\left(x_{a}\right) \\
& F_{b}=-A_{T} T_{1}\left(x_{b}\right)
\end{aligned}
$$

Thus, replacing Equation (20) into Equations (21) and (22) and considering the mechanical impedance of the transducer given by

$$
Z_{T}=A_{T} Z_{T}^{A}=\frac{k}{\omega s_{11}} A_{T}
$$

the forces $F_{a}$ and $F_{b}$ can be obtained as follows

$$
\begin{aligned}
& F_{a}=Z_{T}\left(m \cdot e^{-j k x_{a}}-n \cdot e^{j k x_{a}}\right)-\mathrm{j} \frac{d_{31}}{s_{11} \varepsilon_{33}} \frac{A_{T}}{\omega A_{E}} I_{C} \\
& F_{b}=Z_{T}\left(m \cdot e^{-j k x_{b}}-n \cdot e^{j k x_{b}}\right)-\mathrm{j} \frac{d_{31}}{s_{11} \varepsilon_{33}} \frac{A_{T}}{\omega A_{E}} I_{C}
\end{aligned}
$$

The velocities $v_{a}$ and $v_{b}$ that reach the sides of the transducer with coordinates $x_{a}$ and $x_{b}$, respectively, are given by

$$
\begin{gathered}
v_{a}=v\left(x_{a}\right)=m \cdot e^{-j k x_{a}}+n \cdot e^{j k x_{a}} \\
v_{b}=-v\left(x_{b}\right)=-m \cdot e^{-j k x_{b}}-n \cdot e^{j k x_{b}}
\end{gathered}
$$

Considering the trigonometric identify $2 j \sin (\theta)=e^{j \theta}-e^{-j \theta}$ and the relation $x_{b}-x_{a}=\ell$, as shown in Figure 2, the terms $m$ and $n$ in Equations (26) and (27) can be computed as follows

$$
\begin{gathered}
m=\frac{v_{a} e^{j k x_{b}}+v_{b} e^{j k x_{a}}}{2 j \sin (k \ell)} \\
n=-\frac{v_{a} e^{-j k x_{b}}+v_{b} e^{-j k x_{a}}}{2 j \sin (k \ell)}
\end{gathered}
$$


Replacing Equations (28) and (29) into Equations (24) and (25) and considering the trigonometric identify $2 \cos (\theta)=e^{j \theta}+e^{-j \theta}$, the expressions for the forces $F_{a}$ and $F_{b}$ can be rewritten as

$$
\begin{aligned}
& F_{a}=Z_{T}\left(\frac{v_{a}}{j \tan (k \ell)}+\frac{v_{b}}{j \sin (k \ell)}\right)-\mathrm{j} \frac{d_{31}}{s_{11} \varepsilon_{33}} \frac{A_{T}}{\omega A_{E}} I_{C} \\
& F_{b}=Z_{T}\left(\frac{v_{a}}{j \sin (k \ell)}+\frac{v_{b}}{j \tan (k \ell)}\right)-\mathrm{j} \frac{d_{31}}{s_{11} \varepsilon_{33}} \frac{A_{T}}{\omega A_{E}} I_{C}
\end{aligned}
$$

We need to determine the total current, which is the response of the transducer due to changes in the mechanical properties of the monitored structure. The total current can be obtained from the electric displacement.

The electric displacement in Equation (4) can be rewritten as

$$
D_{3}=\frac{d_{31}}{s_{11}} \frac{\partial u_{x}}{\partial x}+\varepsilon_{33} E_{3}
$$

The electric charge $Q$ can be obtained from the electric displacement integrating Equation (32) with respect to area of the electrodes

$$
Q=\int_{S} D_{3} d s=\varepsilon_{33} E_{3} A_{E}+\frac{d_{31}}{s_{11}} \ell\left[u\left(x_{b}\right)-u\left(x_{a}\right)\right]
$$

Since the PZT patch is very thin, the electric field is practically constant in the z-axis direction and it can be calculated as follows

$$
E_{3}=\frac{U}{t}
$$

In addition, the static capacitance $C_{0}$ of the patch is given by

$$
C_{0}=\varepsilon_{33} \frac{A_{E}}{t}
$$

Substituting Equations (34) and (35) into Equation (33), we obtain

$$
Q=C_{0} U+\frac{d_{31}}{s_{11}} \ell\left[u\left(x_{b}\right)-u\left(x_{a}\right)\right]
$$

Therefore, the total current $\left(I_{T}\right)$ is obtained differentiating Equation (36) with respect to time, as fallows

$$
I_{T}=\frac{d Q}{d t}=j \omega C_{0} U+\frac{d_{31}}{s_{11}} \ell j \omega\left[u\left(x_{b}\right)-u\left(x_{a}\right)\right]
$$


The velocities $v_{a}$ and $v_{b}$ can be written as a function of the displacements $u\left(x_{a}\right)$ and $u\left(x_{b}\right)$, according to following equations

$$
\begin{gathered}
v_{a}=\frac{\partial}{\partial t} u\left(x_{a}\right)=j \omega u\left(x_{a}\right) \\
v_{b}=-\frac{\partial}{\partial t} u\left(x_{b}\right)=-j \omega u\left(x_{b}\right)
\end{gathered}
$$

From Equations (38) and (39) we obtain

$$
I_{T}=j \omega C_{0} U-\frac{d_{31}}{s_{11}} \ell\left(v_{a}+v_{b}\right)=I_{C}-\frac{d_{31}}{s_{11}} \ell\left(v_{a}+v_{b}\right)
$$

According to Equation (40), besides the current $I_{C}$ due to the capacitance $C_{0}$, there is a current related to the velocities $v_{a}$ and $v_{b}$.

Thus, the voltage $U$ at the terminals of the transducer is given by

$$
U=\frac{d_{31}}{j \omega C_{0} s_{11}} \ell\left(v_{a}+v_{b}\right)+\frac{I_{T}}{j \omega C_{0}}
$$

Or we can also write

$$
U=\frac{I_{C}}{j \omega C_{0}}
$$

Finally, equations (30), (31) and (42) can be rewritten in matrix form, as follows

$$
\left(\begin{array}{l}
F_{a} \\
F_{b} \\
U
\end{array}\right)=-j\left(\begin{array}{ccc}
\frac{Z_{T}}{\tan (k \ell)} & \frac{Z_{T}}{\sin (k \ell)} & \frac{d_{31}}{s_{11} \varepsilon_{33}} \frac{A_{T}}{\omega A_{E}} \\
\frac{Z_{T}}{\sin (k \ell)} & \frac{Z_{T}}{\tan (k \ell)} & \frac{d_{31}}{s_{11} \varepsilon_{33}} \frac{A_{T}}{\omega A_{E}} \\
0 & 0 & \frac{1}{\omega C_{0}}
\end{array}\right)\left(\begin{array}{l}
v_{a} \\
v_{b} \\
I_{C}
\end{array}\right)
$$

The matrix in Equation (43) is known as the electromechanical impedance matrix and defines the piezoelectric transducer as a hexapole, as shown in Figure 3.

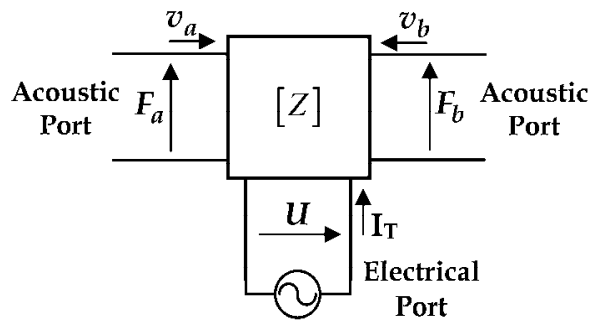

Fig. 3. Piezoelectric transducers can be represented as a hexapole with one electrical port and two acoustic ports. 
According to Figure 3, the transducer is represented by a hexapole with one electrical port and two acoustic ports. Therefore, there is an electromechanical coupling with the monitored structure. Through the acoustic ports the structure is excited so that the dynamic properties can be assessed. Any variation in the dynamic properties of the structure caused by damage changes the mechanical quantities $\left(F_{a}, F_{b}, v_{a}, v_{b}\right)$ and, due to the electromechanical coupling, also changes the electrical quantities $(U, I)$. Therefore, the structural health can be monitored by measuring the current $\left(I_{T}\right)$ and voltage $(U)$ of the transducer.

In practice, the electrical impedance of the transducer is measured. The electrical impedance $\left(Z_{E}\right)$ of the transducer is given by

$$
Z_{E}=\frac{U}{I_{T}}
$$

Thus, it is useful to find an equivalent electromechanical circuit that relates the electrical impedance of the transducer to the mechanical properties of the structure. The equivalent circuit is presented in the next section.

\subsection{Equivalent electromechanical circuit}

An electromechanical circuit makes it easy to analyze the electrical impedance of the transducer in relation to the dynamic properties of the structure, which are directly related to its mechanical impedance. Thus, we should obtain a circuit that establishes a relationship between the electrical impedance of the transducer and the mechanical impedance of the host structure.

Given the following trigonometric identify

$$
\frac{1}{\tan (k \ell)}=\frac{1}{\sin (k \ell)}-\tan \left(\frac{k \ell}{2}\right)
$$

And considering the following manipulation

$$
-j \frac{A_{T}}{\omega \varepsilon_{33} A_{E}} I_{C}=\ell \frac{t}{j \omega \varepsilon_{33} A_{E}} I_{C}=\ell \frac{1}{j \omega C_{0}} I_{C}=\ell U
$$

We can rewrite Equations (30) and (31) as follows

$$
\begin{aligned}
& F_{a}=-j \frac{Z_{T}}{\sin (k \ell)}\left(v_{a}+v_{b}\right)+j Z_{T} \tan \left(\frac{k \ell}{2}\right) v_{a}+\frac{d_{31}}{s_{11}} \ell U \\
& F_{b}=-j \frac{Z_{T}}{\sin (k \ell)}\left(v_{a}+v_{b}\right)+j Z_{T} \tan \left(\frac{k \ell}{2}\right) v_{b}+\frac{d_{31}}{s_{11}} \ell U
\end{aligned}
$$

From Equations (41), (42), (47), and (48), we can easily obtain the circuit shown in Figure 4 (a). The mechanical and electrical quantities are related through the electromechanical transformer with ratio (TR) given by 


$$
T R=\frac{d_{31}}{s_{11}} \ell
$$

The circuit in Figure 4 (a) is not suitable for analysis of structural damage detection because it does not consider the monitored structure as a propagation media in each acoustic port of the transducer. Both the sides of the circuit, which corresponds to the acoustic ports, must be loaded by the mechanical impedance of the structure, as shown in Figure 4 (b).

The mechanical impedance $\left(Z_{S}\right)$ is given by (Kossoff, 1966)

$$
Z_{S}=A_{S}\left[\frac{\rho_{S} v}{1+\gamma^{2}}+j \frac{\gamma \rho_{S} v}{1+\gamma^{2}}\right]
$$

where $\rho_{S}$ is the mass density of the structure, $A_{S}$ is the cross-sectional area of the structure, as shown in Figure 2, orthogonal to the wave propagating at velocity $v$ and $\gamma$ is the damping, i.e., the loss factor in nepers.
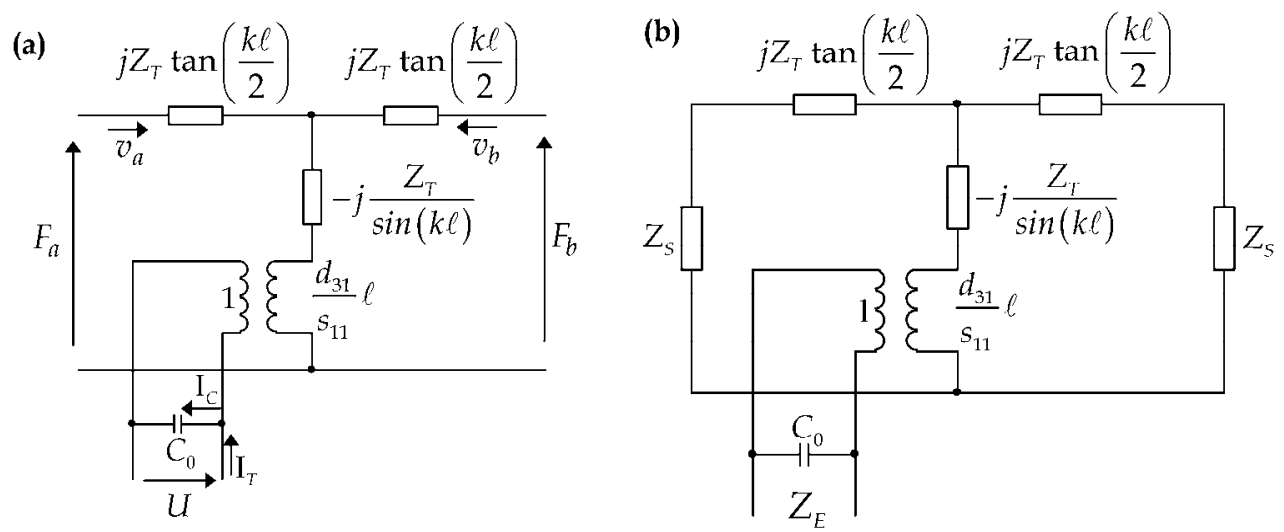

Fig. 4. (a) Piezoelectric transducer represented by an equivalent electromechanical circuit and (b) both acoustic ports loaded by the mechanical impedance of the host structure.

Analyzing the circuit in Figure 4 (b), we can obtain the equivalent electrical impedance between the terminals of the transducer, which is given by

$$
Z_{E}=\frac{1}{j \omega C_{0}} \| j Z_{T}\left(\frac{s_{11}}{d_{31} \ell}\right)^{2}\left[\frac{1}{2} \tan \left(\frac{k \ell}{2}\right)-\frac{1}{\sin (k \ell)}+\frac{Z_{S}}{j 2 Z_{T}}\right]
$$

According to Equation (51), there is a relationship between the mechanical impedance of the monitored structure and the electrical impedance of the piezoelectric transducer. Changes in the mechanical impedance of the structure due to damage result in a corresponding change in the electrical impedance of the transducer. Therefore, structural damage can be characterized by measuring the electrical impedance in an appropriate frequency range. This is the basic principle of damage detection discussed in the next section. 


\subsection{Damage detection}

The comparison between the electrical impedance signatures of a PZT transducer unbonded and bonded to an aluminum beam in a frequency range of $10-40 \mathrm{kHz}$ is shown in Figure 5 . When the transducer is bonded to the structure, several peaks are observed in both the real part and the imaginary part signatures.
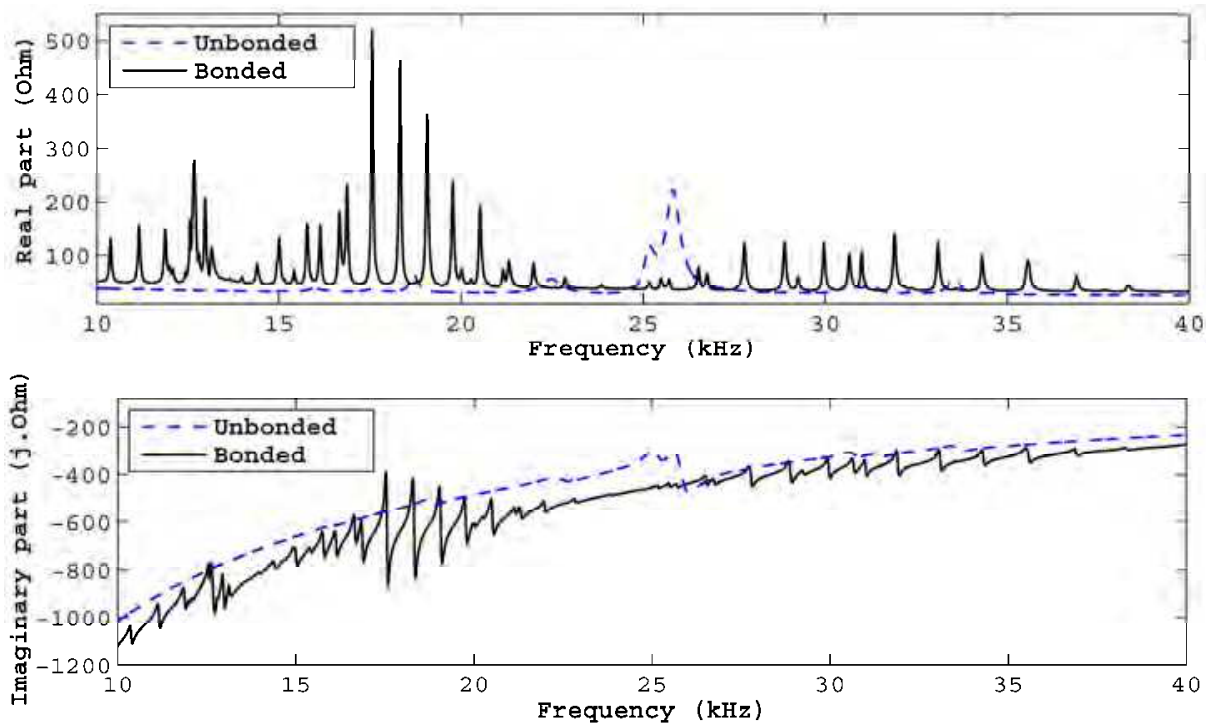

Fig. 5. Real part and imaginary part of the electrical impedance signatures in a frequency range of $10-40 \mathrm{kHz}$ for a PZT transducer unbonded and bonded to an aluminum beam.

These peaks are related to the natural frequencies of the monitored structure. Changes in the natural frequencies either in frequency shifts or variations in the amplitude may indicate structural damage.

Usually, the characterization of damage is performed through metric indices by comparing two impedance signatures, where one of these is previously acquired when the structure is considered healthy and used as reference, commonly called the baseline. Thus, the electrical impedance is repetitively acquired and compared with the baseline signature.

Various indices have been proposed in the literature for damage detection, but the most widely used is the root mean square deviation (RMSD) which is based on Euclidian norm (Giurgiutiu \& Rogers, 1998). Some changes in this index have been suggested by several researchers. One of the most used is given by

$$
R M S D=\sum_{n}^{M} \sqrt{\frac{\left(Z_{n, d}-Z_{n, h}\right)^{2}}{Z_{n, h}^{2}}}
$$

where $Z_{n, h}$ and $Z_{n, d}$ are the electrical impedance (magnitude, real or imaginary part) for the host structure in healthy and damaged condition, respectively, measured at frequency $n$, 
and $M$ is the total number of frequency components, which is related to the frequency resolution of the measurement system.

The index in Equation (52) should be calculated within an appropriate frequency range, which provides good sensitivity for damage detection. Generally, the suitable frequency range is selected experimentally by trial and error methods, but recently some researchers have proposed more efficient methodologies (Peairs et al., 2007; Baptista \& Vieira Filho, 2010). In addition to selecting the appropriate frequency range, it is essential that the measurement system has a good sensitivity and repeatability to avoid either false negative or false positive diagnosis in detecting damage. The measurement systems based on virtual instrumentation are presented in the next section.

\section{Electrical impedance measurement}

Normally, the measurement of the electrical impedance, which is the basic stage of the EMI technique, is performed by commercial impedance analyzers such as the 4192A and 4294A from Hewlett Packard / Agilent, for example. Besides the high costs, these instruments are slow, making it difficult to use the technique in real-world applications, where it is required to use multiple sensors and to diagnose the structure in real-time. The conventional impedance analyzers use a pure sinusoidal wave at each frequency step, making a stepwise measurement under steady-state condition within an appropriate frequency range. Based on this principle, many researchers have developed alternative and low-cost systems for general impedance measurements. Usually, these systems are based on the volt-ampere method (Ramos et al., 2009) where the sinusoidal signal at each frequency step is supplied by a function generator or a direct digital synthesizer - DDS (Radil et al., 2008).

Steady-state measurement systems for specific applications in SHM have also been proposed. In the system proposed by Panigrahi et al. (2010), a function generator was used to excite gradually the piezoelectric transducer with pure sinusoidal signals at each frequency step and an oscilloscope was employed to measure the output response at each excitation frequency. This system is an improvement from a previous work developed by Peairs et al., (2004) where a fast Fourier transform (FFT) analyzer was used to obtain the electrical impedance in the frequency domain. Recently, Analog Devices developed a miniaturized high precision impedance converter, which includes a frequency generator, a DDS core, analog-to-digital converter (ADC) and digital-to-analog converter (DAC), a digital-signal-processor (DSP) integrated in a single chip (AD5933). This chip is used with a microcontroller and other required devices and can provide electrical impedance measurements with high accuracy. This chip has been used in SHM to develop compact and low-cost measurement systems. These new systems support wireless communication and several sensors through analog multiplexer, and can process data locally (Min et al., 2010; Park et al., 2009).

Although steady-state measurement systems provide results with high accuracy, the measurements usually take a long time because the frequency of the pure sinusoidal signal should be gradually increased step-by-step within the suitable range for damage detection. The time consumption may be very significant if a wide frequency range with many steps is required. Accordingly, in these new portable systems a wide frequency range with a narrow frequency step demands a large amount of data that can be difficult to be stored and 
processed locally or transmitted in a wireless mode. Moreover, these systems are difficult to assemble or require specific evaluation boards, which make them attractive mainly for specific SHM applications. For research and general applications, a simpler system should be developed.

We can design fast and easy to assemble measurement systems if the piezoelectric transducer is excited with a sweep signal and using virtual instrumentation. The concept of virtual instrumentation is shown in Figure 6.

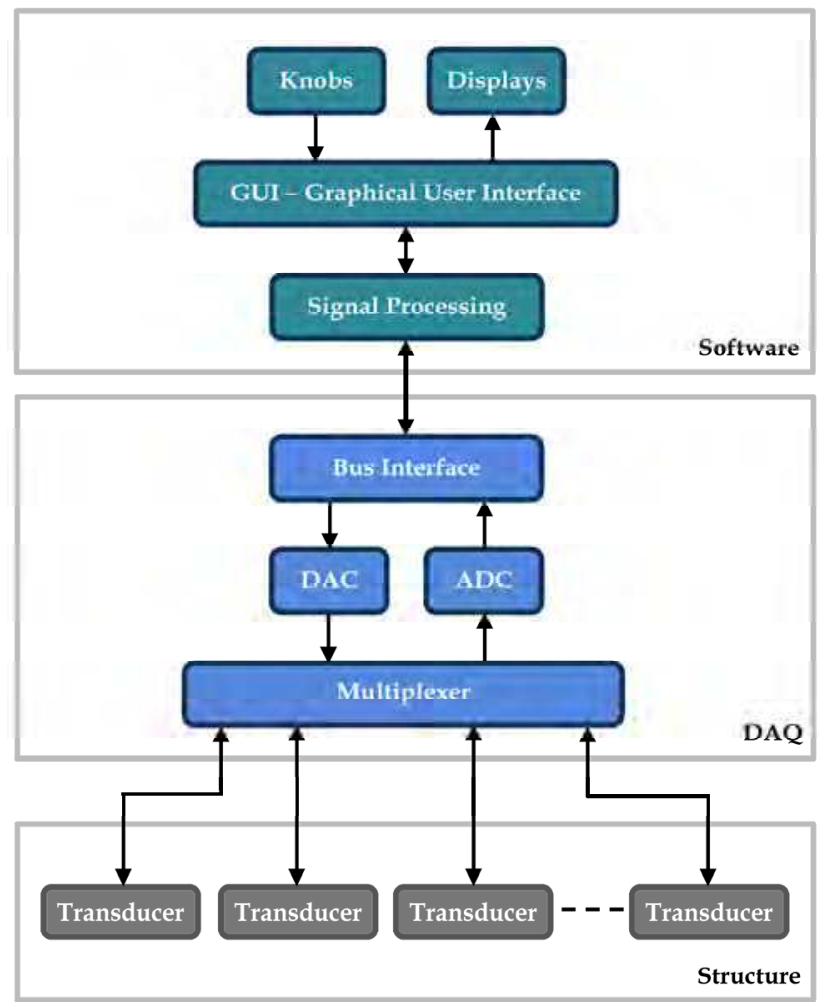

Fig. 6. Data acquisition and virtual instrumentation for structural health monitoring based on EMI methods.

The hardware consists mainly of ADC and DAC converters, multiplexer and bus interface, generally integrated into a single data acquisition (DAQ) device. The DAC generates the excitation signal in a frequency range selected by the user. The response signal of the transducer with information related to the structural health is acquired by the ADC, which must have a sampling rate of at least twice the maximum frequency in the excitation signal. The multiplexer allows the connection of multiple transducers and the bus interface provides the connection with a personal computer.

The control of the DAQ device and the signal processing is done through software. The graphical user interface (GUI) provides knobs and displays for adjusting the parameters of 
the signal generation and acquisition and the visualization of data, such as impedance signatures and metric indices. The virtual instrumentation makes the system very versatile. Adaptations, displays and knobs can be easily added if necessary.

The virtual instrumentation presented in this chapter is mainly based on LabVIEW (Laboratory Virtual Instrument Engineering Workbench), a graphical programming environment from National Instruments. However, other programming platforms can be used, such as Matlab from Mathworks that provides tools for data acquisition and is commonly found in research laboratories.

A basic LabVIEW program for signal acquisition and generation using a DAQ device is shown in Figure 7.

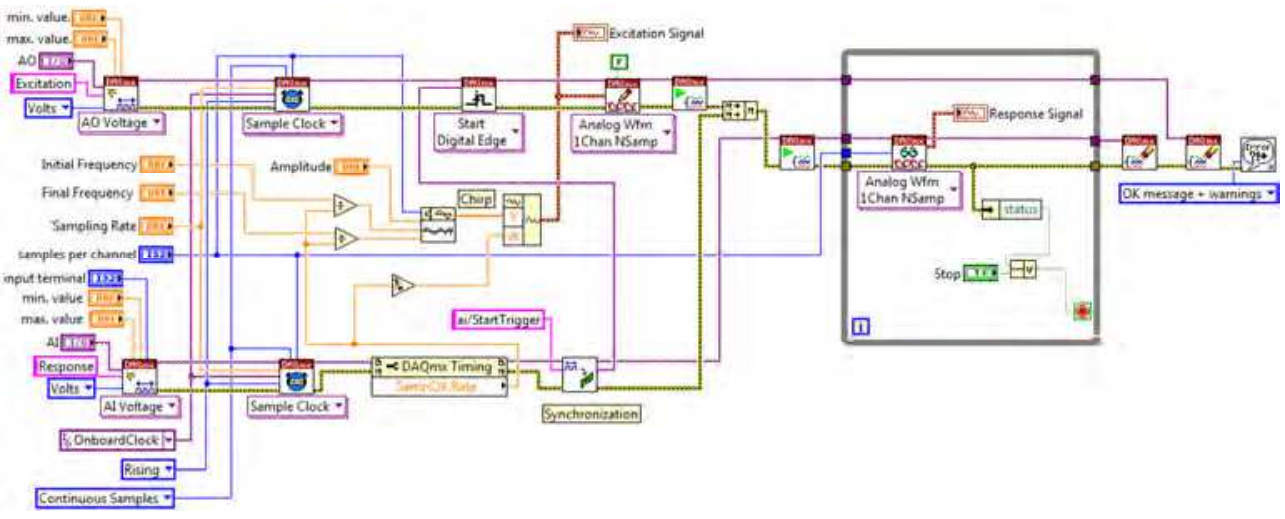

Fig. 7. Basic LabVIEW program for signal acquisition and generation.

The program sets through virtual channels the analog output (AO) and the analog input (AI) used for the excitation of the transducer and the acquisition of the corresponding response signal, respectively. The excitation is performed by a linear chirp signal, which makes a sweep from an initial frequency to a final frequency. The synchronization between the signal generation and acquisition is essential to ensure excellent repeatability between the measurements, which is an important feature in SHM to avoid incorrect diagnosis. The loop structure allows various cycles of excitation and response in applications where is required an average between the measurements for high accuracy.

The characterization of damage can be carried out by analyzing the electrical impedance signatures in the frequency domain or directly the response signal from the transducer in the time domain, as presented in the next sections.

\subsection{Frequency domain analysis}

The analysis in the frequency domain is the usual way to characterize damage and it is usually based on the FRF, as mentioned in the Section 2. The FRF is obtained from the excitation and response signals considered as input and output, respectively, of the system under test containing the structure and the transducer. Thus, from the FRF, we can calculate the electrical impedance in an appropriate frequency range. 
The measurement system proposed by Baptista \& Vieira Filho (2009) is based on the concept shown in Figure 6 and uses a low-cost DAQ device with maximum sampling rate of 250 $\mathrm{kS} / \mathrm{s}$, limiting the impedance measurement up to $125 \mathrm{kHz}$, limiting the impedance measurement up to frequency of $125 \mathrm{kHz}$, although other devices with higher sampling rate can be used without significant changes in the software. The software was developed in LabVIEW with the basic configuration shown in Figure 7. Besides the software, the hardware is very simple and uses only a common resistor in addition to DAQ device to connect the transducer. The system diagram is shown in Figure 8.

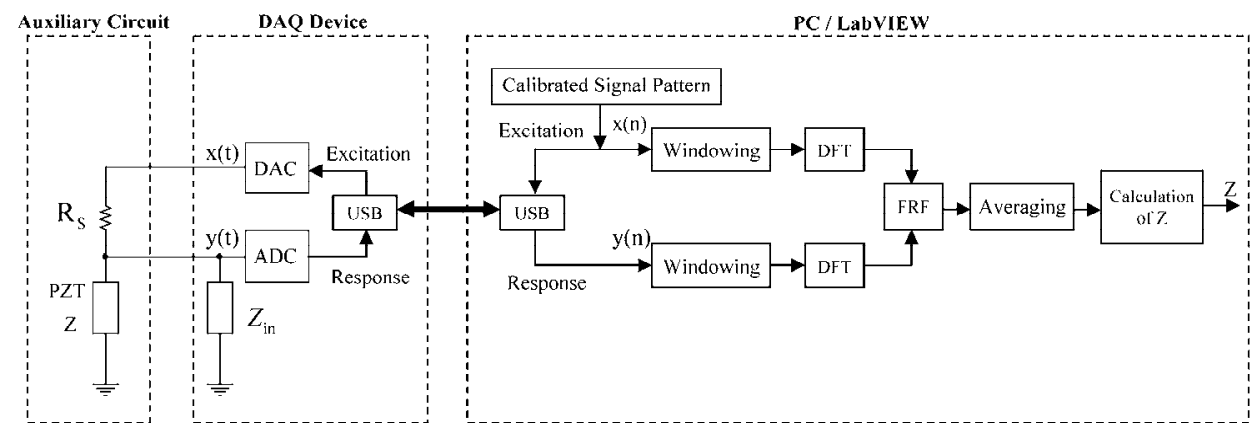

Fig. 8. Example of measurement system based on DAQ device and virtual instrumentation (Baptista \& Vieira Filho, 2009).

In Figure 8, $x(t)$ and $y(t)$ represent the analog signals of excitation and response, respectively, and $x(n)$ and $y(n)$ represent the digital signals. The measurements are based on averaged FRF $(\bar{H})$ obtained from discrete Fourier transform (DFT) of the excitation and response signals. Thus, the electrical impedance $\left(Z_{E}\right)$ in the frequency domain is given by

$$
Z_{E}[n]=\frac{\overline{H[n]} \cdot R_{S}\left(r+Z_{\text {in }}[n]\right)}{Z_{\text {in }}[\mathrm{n}]-\overline{H[n]}\left(R_{S}+r+Z_{\text {in }}[\mathrm{n}]\right)}
$$

where the resistor $R_{S}$ is a current limiter, $r$ is the resistance of the cable used to connect the DAQ device and the PZT sensor, and $Z_{i n}$ is the input impedance of the DAQ device and $n$ is de frequency of the chirp signal ranging from an initial low value to a final high value as defined in the basic program shown in Figure 7.

This system was tested in a PZT transducer bonded to an aluminum beam and the results were compared with the measurements obtained using a conventional impedance analyzer 4192A from Hewlett Packard. The comparison between the two electrical impedance signatures in a frequency range of $30-50 \mathrm{kHz}$ is shown in Figure 9. The similarity between the two signatures indicates the accuracy and feasibility of the system based on virtual instrumentation. The discrepancy between the two measures is less than $4 \%$. Besides the good accuracy, the measurement system provides fast measurements, versatility and lowcost compared to the conventional impedance analyzers.

Improvements such as multiple sensors and real-time diagnosis can be easily included. The system diagram shown in Figure 10 has these features (Baptista et al., 2011). As in the 
previous system, the excitation signal $x(t)$ is generated by the DAC which has a output impedance $R_{\text {out }}$ and the corresponding response signal $y(t)$ from each PZT sensor is acquired by the ADC which has a input impedance $Z_{I N}$ constituted by a high resistance connected in parallel with a capacitance. The low cost DAQ devices have only one ADC and each analog input is routed through an onboard multiplexer (MUX). The samples $n$ of the excitation and the response signals in the discrete form $(x[n]$ and $y[n])$ are synchronized by software through the onboard clock.

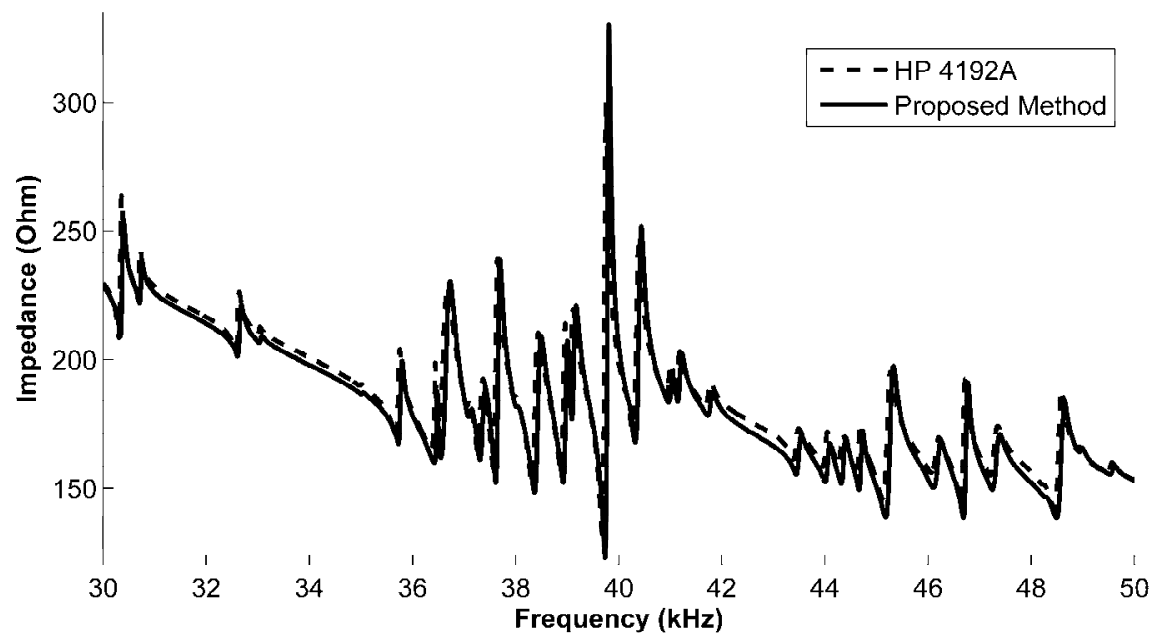

Fig. 9. Comparison between the electrical impedance signatures obtained with a conventional impedance analyzer and the system based on virtual instrumentation.

The resistors $R_{L}$ are current limiters and are connected in series with the sensors. The sensors have common ground, which facilitates the installation of multiple sensors in metallic structures. This common ground should be connected to the DAQ ground.

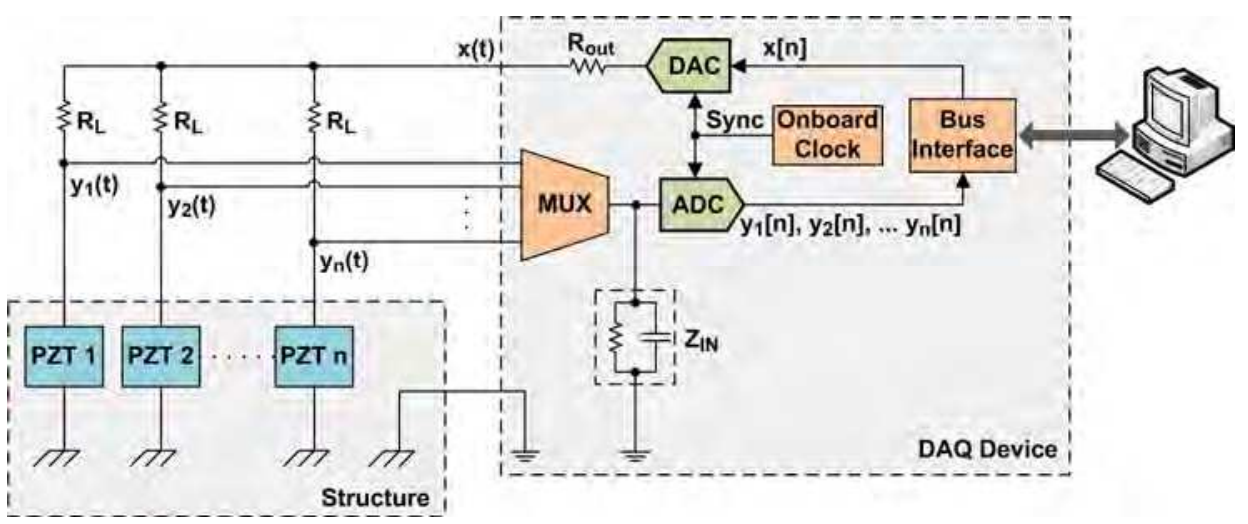

Fig. 10. Improved measurement system including multiple sensors and real-time diagnosis (Baptista et al., 2011). 
Typically, the output impedance $R_{\text {out }}$ is very low on the order of tenths of Ohm. If the resistances $R_{L}$ are much higher than the output impedance, i.e., $R_{L}>R_{\text {out }}$, the excitation signal $x(t)$ in the analog output of the DAQ device can be considered constant in relation to the variations in the electrical impedance of the sensors. Consequently, any mutual interference between the acquisition channels can be neglected and the variations in the response signals $y_{1}(t), y_{2}(t), \ldots, y_{n}(t)$ from each sensor are caused only by the mechanical properties of the host structure or other environmental conditions. The piezoelectric transducers, especially those made of thin PZT ceramics, require low voltage and low current for the excitation signal, so that the piezoelectric effect is linear (Sun et al., 1995; Baptista et al., 2010). Thus, the resistance $R_{L}$ may be on the order of some thousands of Ohm and the condition $R_{L}>R_{\text {out }}$ is easily satisfied.

As in the previous system, the software was implemented in LabVIEW. Figure 11 shows a user-friendly interface that allows the adjustment of the parameters of the signal generation and signal acquisition. A display shows the baseline and the current impedance signatures of the selected sensor and vertical bars indicate the level of damage. In addition, a panel displays a 3D model of the host structure, a LabVIEW tool known as sensor mapping. In this 3D model, virtual sensors are placed in the same positions that they have in the real-world structure, changing the color of the model according to the intensity and in the regions indicated by the metric indices. This feature gives a reasonable suggestion of the damage location, especially in large structures with many sensors. Note instead of the RMDD index, it was used the CCDM index, which is based on the correlation coefficient.

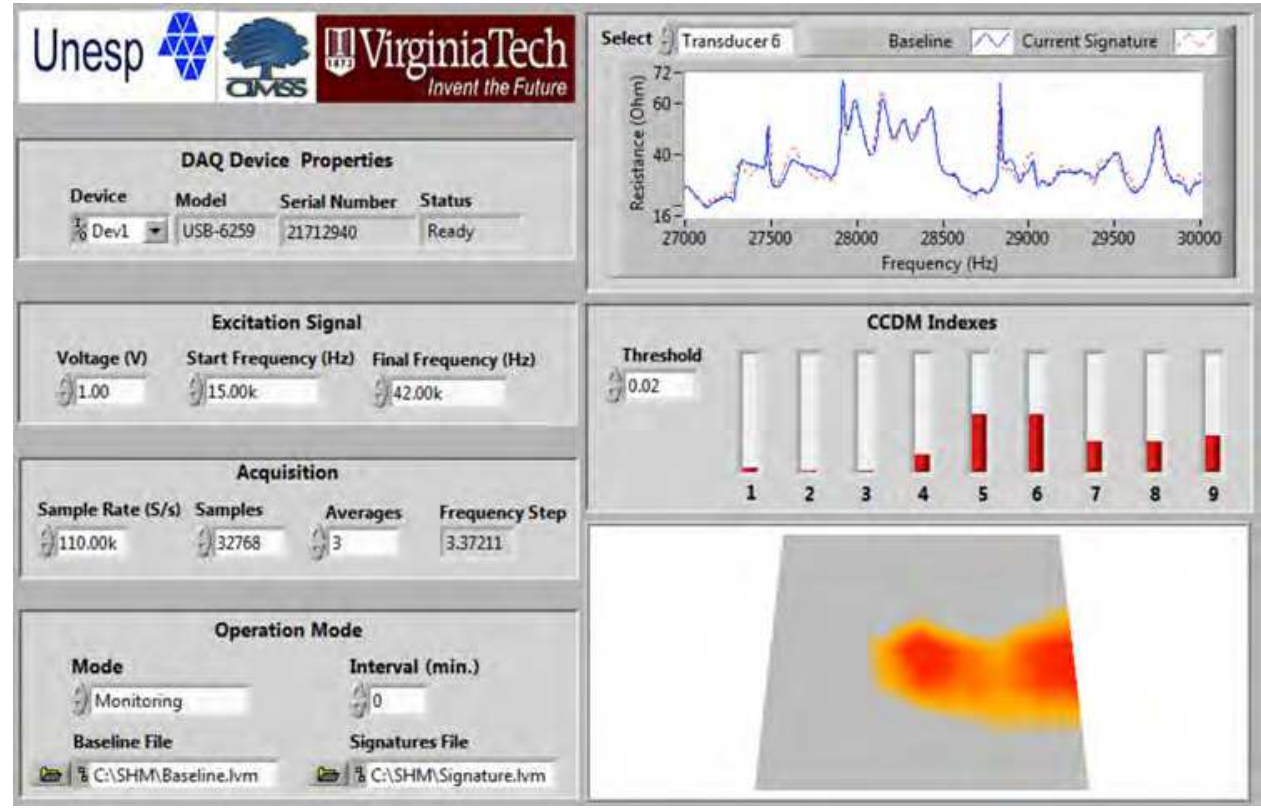

Fig. 11. User-friendly interface of the measurement system (Baptista et al., 2011).

The system was evaluated in an aluminum plate with nine transducers and the DAQ device used was the model USB-6259 from National Instruments. This model has 16 differential 
analog inputs and a maximum sampling rate of $1.00 \mathrm{MS} / \mathrm{s}$ in the aggregated mode. Thus, for the acquisition of the signals from the nine sensors the sampling rate was set to $110 \mathrm{kS} / \mathrm{s}$ with 32768 samples. The results show conclusively that the system has good repeatability, sensitivity to detect damage and provides fast measurements. For this configuration, the measurement of the nine sensors and the presentation of the results are completed in less than 2 seconds in a PC laptop with medium performance.

The systems presented previously are based on the FRF, where structural damage is detected by analyzing the electrical impedance signatures in the frequency domain. However, the characterization of damage can be performed in a simpler method directly in the time domain, as discussed in the next section.

\subsection{Time domain analysis}

In this section, we show that the time response of a piezoelectric transducer provides information on the electromechanical impedance variation when a monitored structure is damaged (Vieira Filho et al., 2011). The time domain approach changes the paradigm of SHM systems based on EMI and the results are similar to those obtained using electrical impedance measurements in the frequency domain. The efficiency of the time domain approach was demonstrated through experiments using an aluminum plate. The results using both the FRF and the time response were obtained and compared.

In the time domain approach, the analysis of only the time response $y(t)$ of the transducer using the system presented in Figure 8 is sufficient to detect damage. From Figure 8, the time response of the transducer $y(t)$ in relation to the excitation signal $x(t)$ can be obtained through an inverse Fourier or Laplace transform according to the following equation

$$
Y=\frac{Z_{E}}{Z_{E}+R_{S}} X
$$

where $Z_{E}$ is the electrical impedance of the transducer.

From a practical point of view, the inverse transform is not necessary because the time response is directly obtained. However, the response signal $y(t)$ changes according to the electrical impedance $Z_{E}$ and the input signal $x(t)$. Considering an input signal with constant amplitude and frequency, the response signal $y(t)$ will change only if the electrical impedance $Z_{E}$ changes, which according to Equation (51) occurs when the structure suffers any type of damage and its mechanical impedance $Z_{S}$ changes. In this case, the signal $y(t)$ could be directly related to the health condition of the monitored structure. If $x(t)$ is a pure sine wave signal with peak amplitude $V_{p x}$ and fixed frequency $\omega_{x}$, it can be shown (Radil et al., 2008) that the approximate magnitude of the electrical impedance $Z_{E}$ is given by

$$
\left|Z_{E}\right| \cong \frac{V_{p y}}{V_{p x}-V_{p y}} R_{S}
$$

where $V_{p y}$ represents the amplitude peak of the response signal $y(t)$.

For a complete characterization of $Z_{E}$, it is possible to compute both the real and the imaginary parts. This is quite direct if the phase difference between $x(t)$ and $y(t)$ is known. 
However, the magnitude of the impedance might assure good sensitivity as well and this will be shown in the example results. So, considering a constant peak value of $x(t)$, the response $y(t)$ will be modified according to any variation of $Z_{E}$, which affirms that $y(t)$ is function of $Z_{E}$. This approach is enough to detect damage because it is sensitive to any structural change. Furthermore, an efficient SHM method based on the EMI does not have to measure the electrical impedance itself, but just measure its variation. This new methodology is called here the time electrical impedance (TEI).

An experiment using an aluminum plate of $500 \times 300 \times 2 \mathrm{~mm}$ with four PZT transducers was carried out to validate the TEI method (Vieira Filho et al., 2011). Figure 12 presents the time response signal $y(t)$ in both healthy and damage conditions.

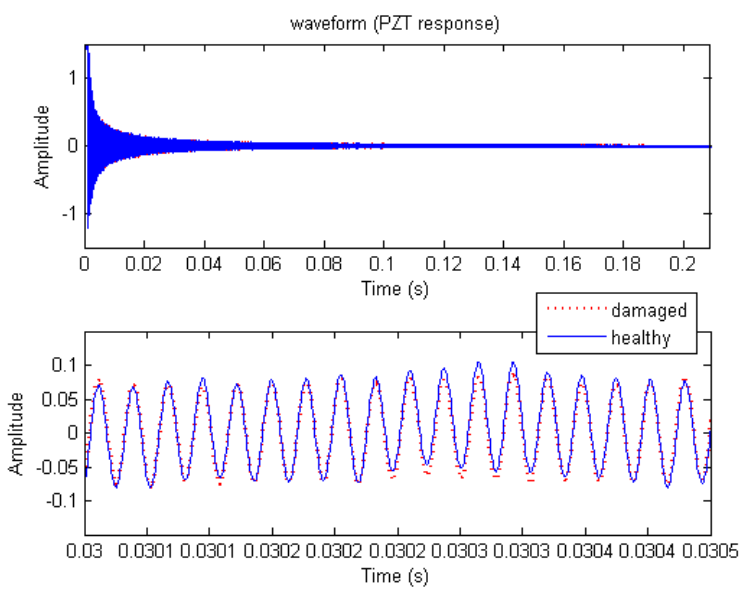

Fig. 12. Time response signal $y(t)$ in health and damage conditions.

We can observe that both responses are close. However, a significant difference can be observed if a subtraction between time responses $\left(y_{b}[n]-y_{m}[n]\right)$ is carried out, where $y_{b}$ is the baseline, $y_{m}$ is the updated time response and $n$ is the sample. This operation was carried out first between the baseline and the time response in the healthy condition and then between the baseline and the time response in the damaged condition. The results are presented in Figure 13 and they show that this operation gives suitable information on the structure's condition. As a result, it is expected that these differences could be detected using metric indices, such as RMSD.

The TEI method was implemented and the results were compared to the ones obtained using the traditional EMI based on the FRF. Thus, the RMSD values were obtained for both TEI and FRF. It is important to observe that although the absolute values of the indices are not the best method for comparing the results, they are interesting for the purpose of evaluating each method separately. However, since the goal is to compare TEI and FRF, the indices are presented using the ones obtained in healthy condition as reference (called normalized here). Figure 14 shows the normalized RMSD values for (a) TEI and (b) for FRF obtained using a plate with four PZT patches and damage simulated at three different positions. According to the experimental results, the RMSD values obtained in the time 
domain are significantly higher than those obtained from FRF. In the time domain, the variation in these values for the damaged structure in relation to the healthy condition reaches a factor of 45 times greater. On the other hand, in the frequency domain this variation is only about 12 times greater.
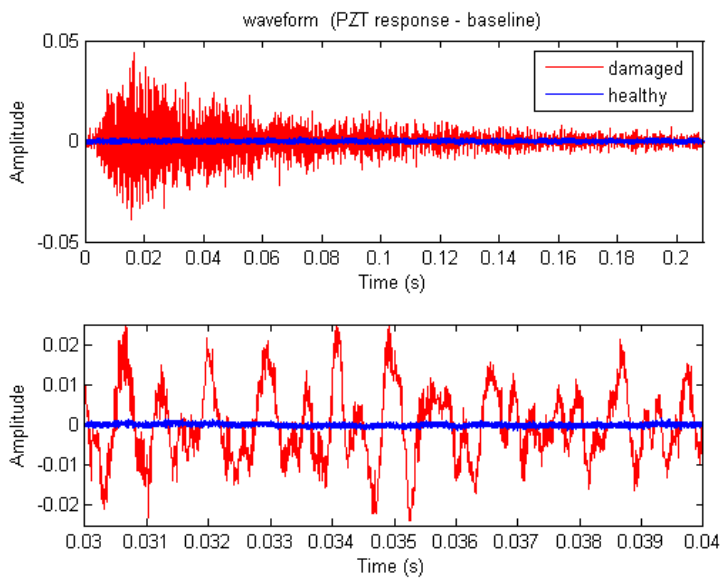

Fig. 13. Difference between the baseline and updated time response signals.

(a)

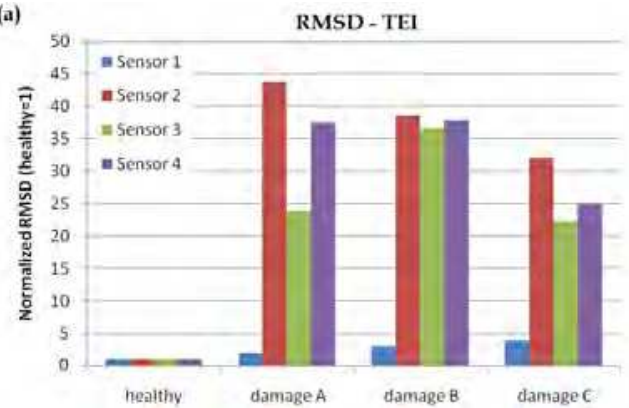

(b)

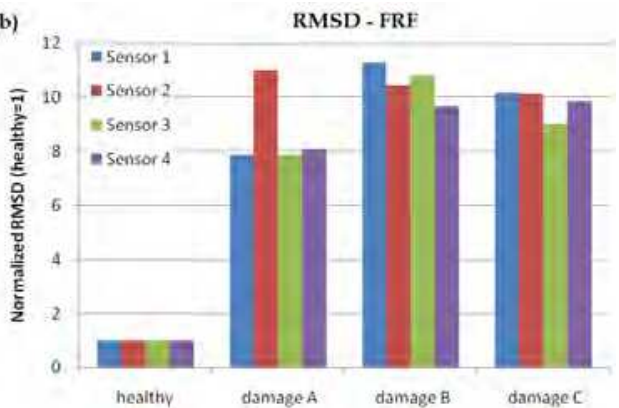

Fig. 14. Normalized RMSD values for (a) TEI and (b) for conventional FRF method.

Therefore, the results indicate conclusively that the characterization of damage in the time domain is feasible and has excellent sensitivity. Since the analysis is carried out directly from the time response signal and it is not required to compute the DFT, the TEI is simpler than the conventional EMI method.

\section{Conclusion}

In this chapter, we have presented the basic principle of the electromechanical impedance technique for detecting damage in structural health monitoring. The measurement of the electrical impedance of piezoelectric transducers, which is the basic stage of the technique, was addressed focusing the virtual instrumentation. The analysis in the frequency domain and the time domain were presented. The experimental results show conclusively that the 
measurement systems based on virtual instrumentation are feasible and efficient for both methods of analysis.

It is important to note that the equivalent electromechanical circuit and the experimental results presented here were obtained for the PZT ceramics. However, these ceramics are brittle and in some applications it is advantageous to use MFC transducers, which are more flexible. In addition, the electromechanical impedance signatures are significantly sensitive to temperature variations. Therefore, the measurements systems should include some compensation method to correct the changes in the impedance signatures for practical applications under temperature variations.

\section{Acknowledgment}

The authors would like to thank the Center for Intelligent Material Systems and Structures, Virginia Tech, and INCT-EIE. This work was partially supported by the Capes Foundation, Ministry of Education of Brazil, grant numbers BEX 0125/10-5 and BEX 3634/09-4.

\section{References}

Baptista, F.G. \& Vieira Filho, J. (2009). A New Impedance Measurement System for PZT Based Structural Health Monitoring. IEEE Transactions on Instrumentation and Measurement, Vol. 58, No. 10, (October 2009), pp. 3602-3608, ISSN 0018-9456

Baptista, F.G. \& Vieira Filho, J. (2010). Optimal Frequency Range Selection for PZT Transducers in Impedance-Based SHM Systems. IEEE Sensors Journal, Vol. 10, No. 8, (August 2010), pp. 1297-1303, ISSN 1530-437X

Baptista, F.G.; Vieira Filho, J. \& Inman, D.J. (2010). Influence of Excitation Signal on Impedance-Based Structural Health Monitoring. Journal of Intelligent Material Systems and Structures, Vol. 21, No. 14 (November 2010), pp. 1409-1416, ISSN 1045$389 X$

Baptista, F.G.; Vieira Filho, J. \& Inman, D.J. (2011). Real-Time Multi-Sensors Measurement System With Temperature Effects Compensation for Impedance-Based Structural Health Monitoring. Structural Health Monitoring, DOI: 10.1177/1475921711414234 (published online before print), pp. 1-14, ISSN 1475-9217

Cawley, P. (1997). Long Range Inspection of Structures Using Low Frequency Ultrasound, Proceedings of Structural Damage Assessment Using Advanced Signal Processing Procedures, pp. 1-17, University of Sheffield, Sheffield

Giurgiutiu, V. \& Rogers, C.A. (1998). Recent Advancements in the Electro-Mechanical (E/M) Impedance Method for Structural Health Monitoring and NDE, Proceedings of $5^{\text {th }}$ Annual International Symposium on Smart Structures and Materials, Vol.3329, pp. 536547, SPIE, San Diego, USA

Gyekenyesi, A.L.; Martin, R.E.; Sawicki, J.T. \& Baaklini, G.Y. (2005). Damage Assessment of Aerospace Structural Components by Impedance Based Health Monitoring. NASA Technical Memorandum, TM-2005-213579, Hanover, Available from http://gltrs.grc.nasa.gov

Kessler, S.S.; Spearing, S.M.; Atala, M.J.; Cesnik, C.E.S. \& Soutis, C. (2002). Damage Detection in Composite Materials Using Frequency Response Methods. Composites Part B: Engineering, Vol. 33, No. 1, (January 2002), pp. 87-95, ISSN 1359-8368 
Kossoff, G. (1966). The Effects of Backing and Matching on The Performance of Piezoelectric Ceramic Transducers. IEEE Transactions on Sonics and Ultrasonics, Vol. 13, No. 1, (March 1966), pp. 20-30, ISSN 0018-9537

Meitzler, A.H. et al. (1987). IEEE Standard on Piezoelectricity: An American National Standard. Std 176, 66 p., IEEE-ANSI, New York, USA

Min, J.; Park, S.; Yun, C.B. \& Song, B. (2010). Development of Multi-Functional Wireless Impedance Sensor Nodes for Structural Health Monitoring, Proceedings of SPIE Sensors and Smart Structures Technologies for Civil, Mechanical, and Aerospace Systems 2010, vol. 7647, pp. 764728-1-764728-8, San Diego, CA, USA

Panigrahi, R.; Bhalla, S. \& Gupta, A. (2010) A Low-Cost Variant of Electro-Mechanical Impedance (EMI) Technique for Structural Health Monitoring. Experimental Techniques, Vol. 34, No. 2, (March 2010), pp. 25-29, ISSN 1747-1567

Park, S.; Shin, H.H. \& Yun, C.B. (2009) Wireless Impedance Sensor Nodes for Functions of Structural Damage Identification and Sensor Self-Diagnosis. Smart Materials and Structures, Vol. 18, No. 5, (May 2009), pp. 055001, ISSN 0964-1726

Peairs, D.M.; Park, G. \& Inman, D.J. (2004). Improving Accessibility of the Impedance-Based Structural Health Monitoring Method. Journal of Intelligent Material Systems and Structures, Vol. 15, No .2, (February 2004), pp. 129-139, ISSN 1045-389X

Peairs, D.M.; Tarazaga, P.A. \& Inman, D.J. (2007). Frequency Range Selection for Impedance-Based Structural Health Monitoring. Journal of Vibration and Acoustics, Vol. 129, No. 6, (December 2007) pp. 701-719, ISSN 1048-9002

Radil, T.; Ramos, P.M. \& Serra, A.C. (2008). Impedance Measurement with Sine-Fitting Algorithms Implemented in a DSP Portable Device. IEEE Transactions on Instrumentation and Measurement, Vol. 57, No. 1, (January 2008), pp. 197-204, ISSN 0018-9456

Ramos, P.M.; Janeiro, F.M.; Tlemçani, M. \& Serra, A.C. (2009). Recent Developments on Impedance Measurements with DSP-Based Ellipse-Fitting Algorithms. IEEE Transactions on Instrumentation and Measurement, Vol. 58 No. 5, (May 2009) pp. 16801689, ISSN 0018-9456

Royer, D. \& Dieulesaint, E. (2000). Elastic Waves in Solids II: Generation, Acousto-Optic Interaction, Applications, Vol. 2, 446 p., Springer, Berlin

Rytter, A. (1993). Vibration Based Inspection of Civil Engineering Structures. Department of Building Technology and Structural Engineering, Aalborg University, 193 p., Denmark

Sohn, H.; Farrar, C.R.; Hemez, F.M.; Shunk, D.D.; Stinemates, D.W.; Nadler, B.R. \& Czarnecki, J.J. (2004). A review of Structural Health Monitoring Literature: 19962001. Los Alamos National Laboratory Report, LA-13976-MS, Available from http:/ / www.lanl.gov

Sun, F.; Chaudhry, Z.; Liang, C. \& Rogers, C.A. (1995). Truss Structure Integrity Identification Using PZT Sensor-Actuator. Journal of Intelligent Material Systems and Structures, Vol. 6, No. 1, (January 1995), pp. 134-139, ISSN 1045-389X

Vieira Filho, J.; Baptista, F.G.; Farmer, J. \& Inman, D.J. (2011). Time-Domain Electromechanical Impedance for Structural Health Monitoring, Proceedings of $8^{\text {th }}$ International Conference on Structural Dynamics, Leuven, Belgium, 4-6 July 2011 
Wang, M.L.; Satpathi, D. \& Heo, G. (1997). Damage Detection of a Model Bridge Using Modal Testing, Proceedings of International Workshop on Structural Health Monitoring, pp. 589-600, DEStech Publications, Stanford, California, USA 


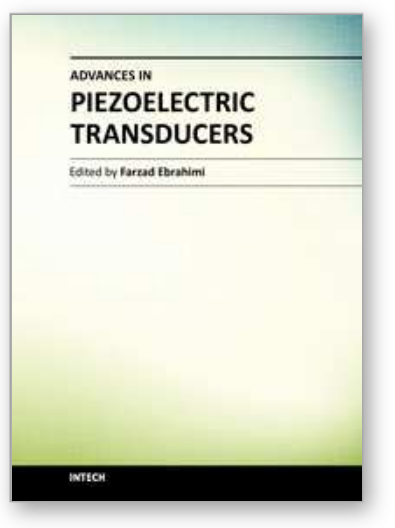

\author{
Advances in Piezoelectric Transducers \\ Edited by Dr. Farzad Ebrahimi
}

ISBN 978-953-307-931-8

Hard cover, 128 pages

Publisher InTech

Published online 25, November, 2011

Published in print edition November, 2011

The piezoelectric transducer converts electric signals into mechanical vibrations or vice versa by utilizing the morphological change of a crystal which occurs on voltage application, or conversely by monitoring the voltage generated by a pressure applied on a crystal. This book reports on the state of the art research and development findings on this very broad matter through original and innovative research studies exhibiting various investigation directions. The present book is a result of contributions of experts from international scientific community working in different aspects of piezoelectric transducers. The text is addressed not only to researchers, but also to professional engineers, students and other experts in a variety of disciplines, both academic and industrial seeking to gain a better understanding of what has been done in the field recently, and what kind of open problems are in this area.

\title{
How to reference
}

In order to correctly reference this scholarly work, feel free to copy and paste the following:

Fabricio Guimarães Baptista and Jozue Vieira Filho (2011). Piezoelectric Transducers Applied in Structural Health Monitoring: Data Acquisition and Virtual Instrumentation for Electromechanical Impedance Technique, Advances in Piezoelectric Transducers, Dr. Farzad Ebrahimi (Ed.), ISBN: 978-953-307-931-8, InTech, Available from: http://www.intechopen.com/books/advances-in-piezoelectric-transducers/piezoelectrictransducers-applied-in-structural-health-monitoring-data-acquisition-and-virtual-instr

\section{INTECH}

open science | open minds

\section{InTech Europe}

University Campus STeP Ri

Slavka Krautzeka 83/A

51000 Rijeka, Croatia

Phone: +385 (51) 770447

Fax: +385 (51) 686166

www.intechopen.com

\section{InTech China}

Unit 405, Office Block, Hotel Equatorial Shanghai

No.65, Yan An Road (West), Shanghai, 200040, China 中国上海市延安西路65号上海国际贵都大饭店办公楼405单元

Phone: +86-21-62489820

Fax: $+86-21-62489821$ 
(C) 2011 The Author(s). Licensee IntechOpen. This is an open access article distributed under the terms of the Creative Commons Attribution 3.0 License, which permits unrestricted use, distribution, and reproduction in any medium, provided the original work is properly cited. 